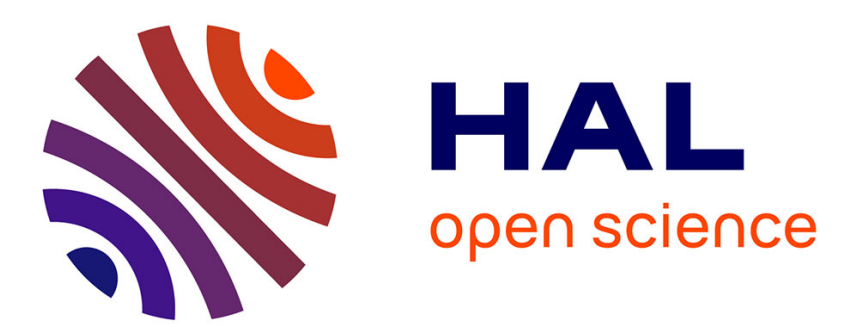

\title{
Généalogie, critique de la responsabilité morale et constitution de la subjectivité selon Nietzsche \\ Thierry Ménissier
}

\section{To cite this version:}

Thierry Ménissier. Généalogie, critique de la responsabilité morale et constitution de la subjectivité selon Nietzsche. Revue des sciences philosophiques et theologiques, 2010, 94 (4), pp.671-689. 10.3917/rspt.944.0671. halshs-01652815

\section{HAL Id: halshs-01652815 https://shs.hal.science/halshs-01652815}

Submitted on 1 Dec 2017

HAL is a multi-disciplinary open access archive for the deposit and dissemination of scientific research documents, whether they are published or not. The documents may come from teaching and research institutions in France or abroad, or from public or private research centers.
L'archive ouverte pluridisciplinaire HAL, est destinée au dépôt et à la diffusion de documents scientifiques de niveau recherche, publiés ou non, émanant des établissements d'enseignement et de recherche français ou étrangers, des laboratoires publics ou privés. 


\title{
GÉNÉALOGIE, CRITIQUE DE LA RESPONSABILITÉ MORALE ET CONSTITUTION DE LA SUBJECTIVITÉ SELON NIETZSCHE
}

\author{
Thierry Ménissier
}

Vrin | Revue des sciences philosophiques et théologiques

\section{0/4 - TOME 94 \\ pages 671 à 689}

ISSN 0035-2209

Article disponible en ligne à l'adresse:

http://www.cairn.info/revue-des-sciences-philosophiques-et-theologiques-2010-4-page-671.htm

Pour citer cet article :

Ménissier Thierry, « Généalogie, critique de la responsabilité morale et constitution de la subjectivité selon Nietzsche »,

Revue des sciences philosophiques et théologiques, 2010/4 TOME 94, p. 671-689. DOI : 10.3917/rspt.944.0671

Distribution électronique Cairn.info pour Vrin.

(C) Vrin. Tous droits réservés pour tous pays.

La reproduction ou représentation de cet article, notamment par photocopie, n'est autorisée que dans les limites des conditions générales d'utilisation du site ou, le cas échéant, des conditions générales de la licence souscrite par votre établissement. Toute autre reproduction ou représentation, en tout ou partie, sous quelque forme et de quelque manière que ce soit, est interdite sauf accord préalable et écrit de l'éditeur, en dehors des cas prévus par la législation en vigueur en France. II est précisé que son stockage dans une base de données est également interdit. 


\title{
GÉNÉALOGIE, CRITIQUE DE LA RESPONSABILITÉ MORALE ET CONSTITUTION DE LA SUBJECTIVITÉ SELON NIETZSCHE
}

\author{
Par Thierry MENISSIER
}

Pour l'éducation nouvelle du genre humain - À l'aide, gens secourables et de bonne volonté, une tâche vous attend : débarrasser le monde du concept de punition qui l'a infesté tout entier! Il n'est pire infection. On n'a pas seulement placé ce concept dans les conséquences de nos actes - et pourtant, quelle monstruosité, quelle déraison il y a déjà à considérer cause et effet comme cause et punition! - on a fait plus et, grâce à l'infâme sophistique du concept de punition, on a entièrement dépossédé de son innocence la pure contingence de ce qui advient ${ }^{1}$.

Dans cet article, nous voulons examiner comment, dans son entreprise de généalogie des valeurs, Nietzsche opère une critique de la notion de responsabilité. Par ce biais, la généalogie apparaît comme un art critique, la responsabilité constituant un de ses objets fondamentaux, mais elle se présente également comme le moyen d'une éthique. La

1. Nietzsche, Aurore. Pensées sur les préjugés moraux, trad. Fr. J. Hervier, Paris, Gallimard, 1980, Livre I, § 13, p. 26. 
critique de la responsabilité fait alors office de moyen privilégié pour renouveler la représentation philosophique des relations intersubjectives, et permet d'engager la réflexion sur la voie d'un nouveau rapport à soi.

\section{IDÉE D’UNE CRITIQUE GÉNÉALOGIQUE DE LA RESPONSABILITÉ}

Dans La généalogie de la Morale, Nietzsche formule son projet dans les termes d'une « exigence nouvelle » :

Nous avons besoin d'une critique des valeurs morales, il faut remettre une bonne fois en question la valeur de ces valeurs elles-mêmes - et pour ce, il faut avoir connaissance des conditions et des circonstances dans lesquelles elles ont poussé, à la faveur desquelles elles se sont développées et déplacées $^{2}$.

L'entreprise généalogique se présente comme un questionnement qui pose les conditions d'un nouveau commencement pour la connaissance morale, dans un geste évocateur de certaines grandes pages qui sont à l'origine de la philosophie moderne ${ }^{3}$. Au lieu d'admettre que les valeurs constituent des données a priori, il est nécessaire d'examiner (1) leurs conditions de possibilité en tant que valeurs (comment il se fait par exemple que l'on puisse hiérarchiser deux types de comportements, en affirmant que tel comportement est moralement préférable à tel autre), (2) la genèse des valeurs qui régissent notre civilisation. Ainsi se comprend le projet généalogique : établir la généalogie de quelqu'un, c'est retrouver tous ses ascendants, et envisager son existence singulière en fonction d'une lignée se développant selon la longue durée, et en regardant de près le déploiement historique des expériences passées afin d'y trouver matière à compréhension de telle existence singulière qui en est l'aboutissement ${ }^{4}$. La généalogie nietzschéenne fait notamment apparaître que nombre de phénomènes moraux et sociaux qui, lorsqu'ils sont envisagés superficiellement, apparaissent comme des causes, ne sont que des effets, ou plus exactement ils ne sont que l'effet des interprétations successives que les hommes ont engendrées sur eux-mêmes. Envisagés

2. La Généalogie de la morale, préface, § 6, trad. fr. P. Wotling, Paris, L.G.F., 2000, p. 56 (le titre complet de l'ouvrage est : Eléments pour la généalogie de la morale. Écrit de combat. Ajouté à Par-delà bien et mal, et publié dernièrement, pour le compléter et l'éclairer).

3. Cf. Descartes, Discours de la Méthode, $\mathrm{II}^{\mathrm{e}}$ partie, dans Euvres philosophiques, Paris, Garnier, 1963, tome I, p. 581 ; et Méditations métaphysiques, Première Méditation, tome II, p. 404.

4. Ici encore, le rapprochement s'impose avec une autre ouvre fondatrice de la philosophie moderne, puisque tel est également, mutatis mutandis, le propos du Discours sur l'origine et les fondements de l'inégalité parmi les hommes de Rousseau. Voir à ce propos la Préface de l'auteur, dans Euvres Complètes, tome III, Paris, Gallimard, 1964, p. $122-127$. 
d'un point de vue métaphysique, ces effets interprétatifs s'enracinent tous dans un principe unique dont l'expression est multiple et déroutante : la volonté de puissance [Wille zur Macht] est cette force structurante qui prend des formes d'une très grande variété, et qui est à la fois la matière indéfinie de l'interprétation et la source qui interprète lorsqu'elle prend la forme de l'humain.

La morale - c'est-à-dire l'ensemble des comportements pour lesquels la conduite se voit assujettie à une règle en fonction d'une hiérarchie de valeurs - se trouve donc elle-même envisagée comme une forme prise par la volonté de puissance. Elle ne renvoie pas à des phénomènes qui seraient par nature moraux : elle constitue une activité qui relève d'une interprétation morale des phénomènes de la volonté de puissance ${ }^{5}$. Cette forme particulière d'expression de la volonté de puissance est délicate à appréhender du fait que grâce à elle, la raison ou le sentiment prétendent se fonder sur leurs propres forces, et révéler des valeurs fondées de toute éternité - dans la morale, la cause réelle des phénomènes est littéralement occultée. De surcroît, si la morale est un symptôme parmi d'autres de la volonté de puissance, on peut affirmer qu'elle en est un symptôme décadent, puisque sa finalité vise à contraindre la volonté de puissance qui est son «support». Elle tend même à dégrader son support, en niant sa force créatrice au nom de valeurs pauvres en possibilités et coercitives. On comprend par là que l'enjeu de la démarche nietzschéenne n'est pas purement intellectuel, car elle a une finalité pratique : elle consiste à lever la chape de plomb que la morale fait peser sur la volonté de puissance, afin de faire surgir de nouveau les riches possibilités de celle-ci.

La responsabilité, quant à elle, ne constitue pas un phénomène moral parmi d'autres ; elle est un phénomène moral d'une importance considérable, puisqu'elle fait fonction de principe pour bien d'autres phénomènes de ce type, et parce que lorsqu'on l'interprète généalogiquement, on voit nettement apparaître le mécanisme même de la morale. Le cheminement suivi par Nietzsche dans le deuxième écrit de la Généalogie promet donc à la fois de donner un aperçu nouveau sur ce phénomène particulier qu'est la responsabilité morale, et de fournir les clefs qui permettent de comprendre d'autres phénomènes du même genre.

L'entreprise généalogique relève quant à elle de l'interprétation historique, en ce qu'elle repose sur la restitution de la constitution des phénomènes moraux. À cet égard, Nietzsche procède en distinguant les différents moments qui furent nécessaires pour faire intégrer par l'animal humain les comportements complexes que recouvre le terme

5. Cf. Par-delà Bien et mal, § 108, trad. fr. C. Heim, Paris, Gallimard, 1971, p. 93 : «Il n'existe pas de phénomènes moraux, mais seulement une interprétation morale des phénomènes ». 
« responsabilité ». Le premier moment de cette intégration semble être la création d'une mémoire, œuvre qui, sur la longue durée, consiste à modifier en profondeur les dispositions corporelles innées d'un animal plutôt porté à oublier ${ }^{6}$. L'homme à qui échoit la responsabilité morale a été dressé pour la supporter et pour ne pouvoir s'en débarrasser ; un lent conditionnement social a été nécessaire, au fil de l'histoire la plus ancienne de l'humanité, afin de réaliser ce double but. Ainsi que Nietzsche l'explique dans le $\S 2$, la moralité des mœurs relève d'un long dressage pulsionnel ${ }^{7}$. Mais c'est surtout la forme même de ce dressage qui permet d'apercevoir les enjeux réels de la responsabilisation morale de l'homme.

\section{LA STRUCTURE DE LA DETTE ET LA « MAUVAISE CONSCIENCE »}

L'homme a été accoutumé à promettre, c'est-à-dire habitué à engager sa parole en affirmant qu'il rendrait coûte que coûte ce qu'on lui a prêté. On lui a donc fait croire avec succès qu'il se devait, afin d'être respectable à ses propres yeux et à ceux des autres, d'honorer demain ses engagements pris aujourd'hui, même si le prix en est exorbitant. Cela revient à aliéner sa liberté future au nom du pacte conclu dans le présent ; le dispositif originel de la responsabilité consiste par suite à enchaîner le futur par le présent. La difficulté d'une telle entreprise - faire acquérir la capacité d'être responsable, cette «puissance sur soi et sur la destinée $»^{8}$ - était extrême, car elle s'appliquait à un être qui par nature se montre particulièrement oublieux. Le $\S 3$ suggère que l'histoire des procédures pénales peut servir de matrice d'interprétation pour comprendre de quelle manière le sentiment d'auto-contrainte a été intériorisé : le spectacle même des châtiments les plus sanglants avait pour fonction de frapper l'imagination afin de provoquer par la peur l'intériorisation du dispositif qui commande de tenir ses promesses. Le sens de l'imputation a été imprimé dans la mémoire de l'animal humain par le biais des modalités les plus cruelles. Mais une fois réussie l'intégration de ce dispositif contre nature, il en est venu à représenter une véritable distinction pour les individus capables de l'être par rapport aux autres. Le paradoxe est que ce qui a été intériorisé excède de loin la capacité de faire face aux conséquences de ses actes : elle consiste à éprouver la «mauvaise conscience », entendue comme l'impression d'être toujours en deçà de

6. Il s'agit alors d' «élever un animal qui soit en droit de promettre », Généalogie de la morale, II, § 1, p. 118.

7. Sur le modèle du dressage du corps comme moyen fondamental de la civilisation, voir Éric BlONDEL, Nietzsche, le corps et la culture, Paris, P.U.F., 1986, et Patrick WoTLING, Nietzsche et le problème de la civilisation, Paris, P.U.F., 1995.

8. Généalogie de la Morale, trad. citée, p. 124. 
ce qu'on doit, ou celle d'être coupable d'une faute qu'aucun effort ne peut venir racheter.

Aussi, le second élément fondamental de l'intégration de la responsabilité est-il révélé par l'analogie qui existe entre la capacité de répondre de ses actes et la structure interindividuelle de la dette ${ }^{9}$. Développer une telle analogie revient à considérer que la matrice de l'intériorisation de la responsabilité se situe en dernière analyse dans un usage social archaïque dont les conditions relatives ont été prises dans l'absolu : il s'agit de la structure contractuelle par laquelle le débiteur s'engageait à s'acquitter de sa dette d'une manière ou d'une autre, le cas échéant en offrant en contrepartie de la somme qu'il ne pouvait verser quelque chose qui est encore en son pouvoir (son corps, celui de quelqu'un de sa famille, son travail, ou sa vie), ou bien qu'il est encore susceptible de perdre bien qu'il ne le possède pas (la béatitude post mortem ou le salut de son âme). Des châtiments physiques et moraux accompagnaient le manquement au contrat, et le fait de pouvoir être cruel dans les formes de la loi, explique Nietzsche, a donné aux créanciers l'impression très satisfaisante de faire partie des maîtres. Schématiquement caractérisée, la notion de responsabilité repose sur une relation spéciale, et d'une grande intensité, qui s'établit à la fois entre les subjectivités (lorsque nous prenons en charge autrui, par exemple dans le cas de la responsabilité parentale) et à l'intérieur de la subjectivité (lorsque nous acceptons les impératifs de la loi ou du devoir, comme dans la responsabilité pénale et la responsabilité morale). Or, l'interprétation fournie par Nietzsche donne à penser que ces deux modalités simplifiées des formes de la responsabilité, à savoir d'une part la capacité de prendre à sa charge le destin de quelqu'un, et de l'autre la faculté de répondre de ses propres actes, ont une racine commune - à savoir, la violence faite à l'individu en ce qui concerne la dette qu'il a à l'égard de quelqu'un d'autre que lui. C'est pourquoi, d'un point de vue nietzschéen, la responsabilité est en réalité inséparable de la culpabilité.

Une telle affirmation peut être entendue sur un mode mineur, comme sur un mode majeur. Mode mineur : la mise en place de la struc-

9. Ibidem, II, § 4, p. 128-131. Ainsi que le rappelle P. WotLING dans son édition de la Généalogie, une telle analogie est d'ailleurs favorisée par la langue allemande, du fait du rapprochement entre Schuld (la faute) et Schulden (les dettes). On peut relever le fait qu'elle est également articulée par des auteurs postérieurs à Nietzsche ; ainsi, Heidegger dans l'importante analyse des $\S \S 58$ à 62 de l'ouvrage Être et temps, met en relation la faute et le «souci »dans l'expérience que le Dasein, cette réalité humaine subjective, fait de lui-même. Cette expérience conjointe lui révèle sa disposition temporelle fondamentale : puisque la faute est un « défaut de » toujours impossible à combler (ce qui est précisément ce qu'éprouve l'être angoissé face au temps), l'analyser en ces termes lui permet d'entendre son rapport fondamentalement ouvert au temps, trad. fr. F. Vezin, Paris, Gallimard, 1986, p. 337-370. 
ture de responsabilité impliquait que, chez l'homme primitif, sensible seulement à la cruauté, le sentiment de culpabilité, bien plus rude que la simple responsabilité, soit imprimé dans sa mémoire, et que de ce fait il apprenne à payer un prix exorbitant dans sa relation à autrui. Mode majeur : la culpabilité est la matrice indépassable de la responsabilité, et toutes les formes de responsabilité se déploient à partir d'elle. Les deux options interprétatives sont possibles. D'une part, en effet, ainsi que l'établit le $\S 8$, la relation débiteur-créancier a fait office d'un schème fondamental par lequel l'homme a appris à se rapporter à autrui, elle est une forme originelle de l'intersubjectivité ; or, dans le même temps, comme la relation induite n'est pas du tout neutre, elle a appris à l'homme à se mesurer à autrui, à s'évaluer soi-même tout en évaluant autrui. Dès le début donc, les liens interpersonnels, sous l'influence de cette maîtresse forme, ont compris une dimension susceptible d'être soupçonnée, du fait qu'ils ont été construits à partir d'une relation de pouvoir visant à rabaisser l'homme primitif, généreux et intempérant, qui, spontanément, déploie sa force de manière surabondante, sans compter. C'est cette générosité même qui cause sa perte, dès lors que la relation dominante devient celle où l'emportent ceux qui comptent étroitement les crédits et les débits.

D'autre part, la seconde interprétation est valide si l'on ne perd pas de vue la dimension métaphysique des phénomènes considérés, à savoir le fait que la responsabilité engage le rapport de l'existence humaine au temps. La base vécue du sentiment de responsabilité consiste à se sentir totalement écrasé par la charge qu'on porte (au point de préférer sa préservation à notre propre vie si un choix de ce genre se présente), de même qu'au sens métaphysique être coupable revient à se percevoir comme perpétuellement débiteur vis-à-vis de quelque chose qu'on ne possède pas et que par définition personne ne peut posséder : l'avenir. C'est la raison pour laquelle la théorie de la responsabilisation prend la forme d'une « histoire du châtiment » qu'il faut entendre à la lumière d'une enquête soupçonneuse à l'égard du travail opéré par la culture sur les individus.

La question posée par Nietzsche, philosophe du soupçon, est en effet : à qui profite une telle structure (comme on le dit d'un crime) ? La réponse qu'il apporte est qu'elle profite à un groupe d'hommes décidés à mettre les autres sous tutelle par le biais des pouvoirs les plus puissants et les plus subtils qui existent, la caste sacerdotale, dont Par-delà bien et mal avait commencé à évoquer le dessein et les ressources. C'est dans cette perspective que se conçoivent les $\S \S 11-15$ de la II ${ }^{e}$ dissertation de la Généalogie: Nietzsche y explique que le système des sanctions et des peines, qu'il nomme du terme générique «le châtiment», a pour fonction d'assujettir radicalement les individualités, moins en les soumettant 
à des peines déterminées au cas par cas, qu'en leur inculquant a priori une culpabilité qui les maintient socialement et moralement soumis à l'ordre dominant. De ce point de vue, Nietzsche s'oppose à toute tentative de justification rationaliste de l'ordre pénal du genre de celle que, dans un esprit proche de la pensée des Lumières, Hegel avait formulée ${ }^{10}$. Ou plus exactement, il interprète cet ordre comme un dispositif qui ne sert pas du tout les fins qu'il prétend servir. Étant un instrument de pouvoir, il n'a pas vocation à permettre la réintégration par la personne jugée d'un ordre civil qui lui permettrait de redevenir digne; il fonctionne comme un système de régulation des pulsions, et, si ce mot a encore un sens dans la doctrine nietzschéenne, loin de contribuer à rehausser la «dignité » de la personne, il la rabaisse fondamentalement, notamment dans l'estime d'elle-même, eu égard à la manière dont elle se représente ses propres forces :

... Il est hors de doute qu'il faille chercher le véritable effet du châtiment avant tout dans un aiguisement de la prudence, dans un allongement de la mémoire, dans une volonté d'opérer à l'avenir avec plus de précaution, plus de méfiance, plus de secret, dans la compréhension du fait que l'on est, une bonne fois pour toutes, trop faible pour bien des choses, dans une espèce d'amélioration du jugement porté sur soi-même. Ce que l'on peut obtenir en gros au moyen du châtiment, chez l'homme et l'animal, c'est l'accroissement de la peur, l'aiguisement de la prudence, la maîtrise des désirs : ce faisant le châtiment dompte l'homme, mais il ne le rend pas « meilleur », - on serait même en droit d'affirmer le contraire avec plus de légitimité encore ${ }^{11}$.

\section{LE DRESSAGE CULTUREL DES SUBJECTIVITÉS}

En procédant de la sorte, le système pénal se présente d'ailleurs comme le symbole même du travail de la culture, ou plus exactement comme le travail d'une certaine culture dont la fin correspond à un tel « domptage $»^{12}$.

10. Voir Hegel, Principes de la philosophie du droit, $\S 100$.

11. Généalogie de la morale, II, 15, trad. citée, p. 162.

12. Cf. ibidem, I, 15, p. 92 : «Ầ supposer que soit vrai ce à quoi l'on croit en tout cas de nos jours comme à une « vérité »: que le sens de toute culture est justement d'élever à partir de la bête de proie « homme » un animal apprivoisé et civilisé, un animal domestique, on devrait alors, sans le moindre doute, considérer tous ces instincts de réaction et de ressentiment grâce auxquels on a fini par briser et subjuguer les lignées nobles avec leurs idéaux comme les authentiques instruments de culture; ce qui ne reviendrait certes pas à dire que du même coup leurs dépositaires représenteraient ainsi par euxmêmes la culture. C'est bien plutôt le contraire qui serait non seulement probable non! qui est aujourd'hui flagrant! Ces dépositaires des instincts d'écrasement altérés de représailles, les descendants de tout le monde d'esclaves européen et non-européen, de toute la population préaryenne en particulier - ils représentent la régression de 
La critique nietzschéenne met en valeur que la responsabilité est comme un corset que l'on fait endosser à la subjectivité, et souligne de quelle manière celle-ci a été accoutumée à le porter, au point d'avoir l'impression qu'elle ne pourrait exister sans lui. Plus exactement, l'interprétation nietzschéenne établit une relation intime entre les formes de la responsabilité juridique (civile et pénale), et la responsabilité morale. Dans le cadre de sa généalogie des phénomènes moraux, l'auteur propose de considérer que ceux-ci sont originairement produits par des dispositifs sociaux destinés à juguler la force des forts; pourtant l'invention et la mise en œuvre de ces dispositifs ont eux-mêmes une origine morale : ils correspondent à une interprétation de la volonté de puissance qui est pleine de ressentiment. Et ces dispositifs ont une finalité morale : pour employer une métaphore que Nietzsche n'aurait peutêtre pas reniée, la responsabilité morale est comme la croix que doit porter la subjectivité. Elle accompagne l'existence qui se transforme de la sorte en chemin du calvaire. L'homme tyrannisé par le devoir a intimement perdu à la fois sa liberté et sa créativité. Le dressage réalisé par la culture du ressentiment n'annule pas purement et simplement les instincts impétueux, elle les intériorise et tourne leur violence contre l'individu :

Tous les instincts qui ne se déchargent pas vers l'extérieur se tournent vers l'intérieur - c'est cela que j'appelle l'intériorisation de l'homme : c'est seulement ainsi que pousse en l'homme ce qu'on appellera ensuite son « âme ». Tout le monde intérieur, originellement mince, comme enserré entre deux peaux, a grossi et est éclos, a gagné en profondeur, en largeur, en hauteur à mesure que la décharge de l'homme vers l'extérieur a été inhibée. Les terribles remparts grâce auxquels l'organisation de l'État se protégeait contre les anciens instincts de liberté - les châtiments font partie au premier chef de ces remparts - produisirent ceci que tous ces instincts de l'homme sauvage, libre, vagabondant se retournèrent, se tournèrent contre l'homme lui-même. L'hostilité, la cruauté, le plaisir pris à la persécution, à l'agression, au changement, à la destruction - tout cela se tournant contre le détenteur de tels instincts : voilà l'origine de la mauvaise conscience ${ }^{13}$.

La «mauvaise conscience »- à savoir, la vie psychique effective de l'homme culpabilisé par la culture - constitue donc le mode subjectif authentique de la conscience morale. Celle-ci n'est autre chose que l'effet d'une discipline, elle apparaît comme une contrainte initialement imposée à la subjectivité et ensuite par elle intériorisée en tant que règle de conduite. Non seulement l'analyse nietzschéenne se trouve donc en totale contradiction avec la thèse kantienne selon laquelle la disposition

l'humanité ! Ces « instruments de culture » sont une honte pour l'homme, et bien plutôt un motif de soupçon, un contre-argument à l'égard de la « culture » en général ! ».

13. Ibidem, II, 16, p. 164. 
à agir selon la loi morale, l'autonomie, manifeste la force de la subjectivité rationnelle; mais encore, elle ouvre la voie à une interprétation renouvelée des techniques permettant d'assujettir l'homme tout en le dotant de l'équipement intellectuel qui fait de lui une subjectivité.

\section{QUELLE FORME POUR LA RESPONSABILITÉ AU-DELÀ DE LA CULPABILITÉ ?}

Si l'on considère à présent le versant non critique de l'entreprise généalogique, son versant éthique - si l'on envisage la généalogie comme le ressort d'un déploiement des possibilités d'existence enfouies dans le passé et toujours potentiellement réactualisables -, la possibilité ouverte par Nietzsche paraît être celle d'une existence dans laquelle la tyrannie de la morale du ressentiment se trouve efficacement combattue. Cela signifie ou bien que la responsabilité morale peut prendre une nouvelle forme, ou bien qu'elle disparaît complètement. Examinons successivement les deux possibilités.

a) La responsabilité peut prendre une nouvelle forme: dans cette perspective, elle se voit dissociée de la culpabilité, et par suite susceptible d'être examinée à nouveaux frais. En tant qu'elle est dissociée de la culpabilité, la responsabilité peut même sembler, à certains égards, rénovée par le travail que lui impose Nietzsche. En effet, ce travail permet à la subjectivité de pouvoir se dire qu'elle n'est pas responsable moralement de tout ce qui lui arrive, et cela lui offre la possibilité de ne pas surcharger exagérément son fardeau pour cheminer dans l'existence. L'examen à nouveaux frais révèle notamment que l'histoire, le cours des choses nous poussent à faire des choix dont nous ne pouvons pas assumer l'entière responsabilité. De ce point de vue, on pourrait dire que l'effet des thèses de Nietzsche ne revient pas à réaliser une déresponsabilisation complète de l'existence, mais plutôt à dissocier, à la fois par principe et comme une méthode de traitement cas par cas, responsabilité et culpabilité.

Paradoxalement, en dépit de tout le mal que Nietzsche pense de cette école de pensée ${ }^{14}$, une telle orientation théorique n'est pas sans évoquer celle prise par les stoïciens, dans leur effort pour séparer, selon les termes du premier paragraphe du Manuel d'Epictète, « ce qui dépend de nous » de «ce qui n'en dépend pas ». À la différence près que tandis que le sage stoïcien, lorsqu'il doit subir l'effet des choses qui ne dépendent pas de lui, reconnaît sur son existence la marque d'un destin tout-puissant, le philologue allemand y voit l'expression de la volonté de puissance, à savoir celle d'une puissance dont les finalités, multiples, complexes et

14. Cf. par exemple Par-delà Bien et Mal, § 9, p. 20-21. 
très difficilement élucidables, mettent en difficulté la tentative de la regarder comme une totalité organisée.

En ce sens, une option que Nietzsche n'aurait peut-être pas démentie consiste à reconnaitre pour la notion de responsabilité une pertinence extra-morale. Plusieurs perspectives sont en réalité ouvertes par cette possibilité. Premièrement, il s'agirait de revendiquer pour l'existence personnelle cette légèreté que seuls peuvent éprouver les enfants, capables de retrouver «l'innocence» de «la pure contingence de ce qui advient », en reprenant les termes de la citation d'Aurore placée en exergue de notre étude. Dès l'ouverture d'Ainsi parlait Zarathoustra, dans le même esprit Nietzsche développe le thème des «trois métamorphoses de l'esprit » : à l'issue de la critique féroce de la culpabilité menée par le «lion» se produit la dernière transformation, et la figure de l'enfant apparait alors ${ }^{15}$. On ne saurait dire de l'enfant qu'il est purement et simplement irresponsable, car son vouloir est actif et se situe audelà de toute contrainte sociale imposée :

Innocence est l'enfant, et un oubli et un recommencement, un jeu, une roue, qui d'elle-même tourne, un mouvement premier, un saint dire Oui.

Oui, pour le jeu de la création mes frères, il est besoin d'un saint dire Oui : c'est son vouloir que veut à présent l'esprit, c'est son monde que conquiert qui au monde est perdu. ${ }^{16}$

Il importe ici de remarquer que la critique de la culpabilité ne ruine pas la possibilité de définir la subjectivité par un certain vouloir. Peutêtre est-ce là une voie d'accès à un nouveau régime de la responsabilité. Une telle manière d'interpréter le travail nietzschéen est confortée par certains passages évoquant la liberté subjective comme capacité de répondre de ses actes ${ }^{17}$.

En second lieu l'exercice de la charge politique, débarrassée de tout préjugé moral - en fonction d'un ethos civique que n'aurait nullement démenti un Machiavel ${ }^{18}$ - peut correspondre à une telle catégorie. Si le philologue allemand n'a pas à proprement parler composé une œuvre de philosophie politique, le réalisme qu'on peut reconnaître à ce grand psychologue qu'était Nietzsche provient notamment de sa capacité à

15. Ainsi parlait Zarathoustra, I, « Des trois métamorphoses », trad. M. de Gandillac, Paris, Gallimard, 1971, p. 35-37.

16. Ibidem, p. 37.

17. Cf. Le crépuscule des idoles, «Flâneries inactuelles », § 38, trad. Ch. Jambet, Paris, Flammarion, p. 156 : «Car, qu'est-ce que la liberté ? C'est avoir la volonté de répondre de soi ».

18. Je me permets de renvoyer le lecteur à mon ouvrage Machiavel, la politique et l'histoire. Enjeux philosophiques, Paris, P.U.F, 2001, particulièrement au chapitre VI : «Une nouvelle norme de vertu : Machiavel critique de l'humanisme civique », p. 165205. 
saisir la logique (littéralement amorale) de l'action politique. Un très grand nombre d'aphorismes témoigne de sa faculté à démasquer les stratégies de puissance qui, au-delà des vertueuses intentions déclarées par les leaders publics, toutes tendances confondues, nourrissent en réalité les projets politiques ${ }^{19}$. Or, cette critique trouve son corrélat pratique dans une forme de lucidité qui apparaissait à Nietzsche comme la condition morale sine qua non de l'esprit politique supérieur. Ainsi vante-t-il les mérites de l'ancienne éthique politique grecque, incarnée dans les figures de Thucydide et de Périclès, modèles de « cette culture de la plus libre connaissance du monde $\gg^{20}$, culture que nous pouvons regarder comme la base d'une forme originale de la responsabilité politique. Le leader, homme affranchi des aspirations humaines ordinaires aussi bien que de toutes les formes de bonnes intentions (même... celles qu'il déclare lui-même afin d'accréditer son action auprès de ses concitoyens), doit en effet composer avec des congénères précisément aveuglés par tout cela. Sa responsabilité s'entend ici comme le fait d'assumer la charge d'un dessein collectif en dépit des contradictions sociales indépassables que connaît tout groupe humain, mais aussi et surtout malgré les limites psychologiques avérées de ses concitoyens.

En troisième lieu, de manière fort différente, peut également correspondre à cette possibilité l'acceptation du registre juridique de la responsabilité personnelle : l'individu qui s'assujettit volontairement aux lois de son pays, sans jamais référer ses motifs d'action à une quelconque moralité, endosse en un certain sens une forme «nietzschéenne » de la responsabilité. Ce qui rend possible une telle interprétation, c'est paradoxalement le fait que Nietzsche a absolutisé la culpabilité de l'être responsable dans le but de dénoncer l'usage moraliste de la notion de responsabilité. Or n'est-ce pas précisément un des enjeux du travail réalisé par le Droit moderne que de distinguer très scrupuleusement l'aire de la responsabilité effective de la culpabilité d'origine psychologique ou superstitieuse ? Par exemple, les différents régimes de la responsabilité civile et pénale et morale reposent sur l'examen précis des circonstances de l'imputation de tel ou tel acte à son auteur ${ }^{21}$. De ce point de vue, l'entreprise juridique donne une continuelle leçon à ce qu'on pourrait nommer « l'esprit de la culpabilité », en tant que le Droit établit rationnellement des responsabilités, après enquête et instruction, ce qui permet

19. Cf. par exemple, dans son intégralité, le troisième Livre d'Aurore.

20. Cf. Aurore, § 168, trad. citée, p. 133. Cf. également Le crépuscule des idoles, «Ce que je dois aux Anciens », § 3 , à propos de la «culture des réalistes [...] - Thucydide comme la vaste somme, la dernière manifestation de ce sens vigoureux, sévère et dur, des réalités, qui était au fond de l'instinct des antiques Hellènes ».

21. Sur ce point, voir par exemple Hans KELSEN, Théorie pure du droit, $2^{\mathrm{e}}$ éd. révisée 1960, les $\S \S 18$ à 23 sur la théorie de l'imputation (trad. Charles Eisenmann [1962], Bruxelles-Paris, Bruylant-LGDJ, 1999, p. 85-108). 
de borner le plus exactement possible les actions dont un prévenu se trouve effectivement responsable et ce dont il se sent coupable ou dont on l'estime coupable. En un sens, le Droit réalise donc l'intention du philologue allemand : si, de son côté, la responsabilité morale est contrainte, la responsabilité juridique repose quant à elle sur les possibilités offertes à la subjectivité d'agir en fonction de droits personnels, puis, le cas échéant, d'être jugée sans que l'on préjuge de sa culpabilité. Par conséquent, la responsabilité extra-morale qui est l'une des perspectives offertes par son œuvre se trouve en partie réalisée par l'exercice multiple du Droit. Ce dernier, ainsi entendu, contribue à construire un «sens commun non substantiel », une forme possible pour la vie commune, relativement neutre par elle-même d'un point de vue axiologique, dans lequel chaque particularité subjective trouve sa place. Ainsi réfléchi, le Droit ne se résume d'ailleurs nullement à une gestion des intérêts et des conflits, mais se comprend à la lumière de sa puissance instituante, ou faculté d'instituer les êtres humains en tant que personnes à partir d'une dogmatique (non axiologique) de leurs relations intersubjectives ${ }^{22}$.

En fin de compte à quoi conduit la thèse nietzschéenne ainsi envisagée ? Elle débouche sur la possibilité offerte à la subjectivité non pas d'être moins responsable, mais d'être plus perspicace quant aux charges qu'elle se donne à elle-même - et de comprendre qu'on lui donne à porter de trop lourds fardeaux. Et quelles sont les ambitions de sa critique radicale de la responsabilité morale? Elle vise à permettre à l'homme de retrouver la liberté créatrice, en inventant une allure nouvelle pour son existence. En ce sens, sans préjuger de la nature de l'ethos par-delà bien et mal, il convient d'affirmer que la philosophie nietzschéenne apparaît comme pleinement éthique, si l'on définit l'éthique comme une réflexion sur les fins que se choisit l'existence.

b) La responsabilité disparaît: on trouve dans la Généalogie de la morale certains passages d'une grande radicalité de ton, qui semblent converger dans ce sens. Ainsi le $\S 13$ du Premier traité, qui part de la tentative de restituer l'interprétation dépitée et haineuse par les tenants de la morale du ressentiment de la « responsabilité » des êtres forts à leur égard :

Que les agneaux soient pénétrés de rancune envers les grands oiseaux de proie, voilà qui n'a rien de surprenant : à ceci près que ce n'est pas une raison pour faire grief aux grands oiseaux de proie de s'emparer de petits agneaux. [...] - Exiger de la vigueur qu'elle ne s'extériorise pas sous forme de vigueur, qu'elle ne soit pas un vouloir-conquérir, un vouloir-subjuguer,

22. Pour une approche du droit dans ces termes, cf. l'ouvrage récent d'Alain SuPIOT, Homo juridicus, Paris, Éditions du Seuil, 2005. 
un vouloir-se-rendre maître, une soif d'ennemis, de résistances et de triomphes, c'est un non-sens exactement au même titre qu'exiger de la faiblesse qu'elle s'extériorise comme vigueur ${ }^{23}$.

Nietzsche se livre alors à une reconstitution de ce qu'est proprement l'expression par les forts de la volonté de puissance :

Un quantum de force est un quantum identique de pulsion, de volonté, de production d'effets - bien plus, ce n'est absolument rien d'autre que justement ce pousser, ce vouloir, cet exercer des effets lui-même, et il ne peut paraître en aller autrement qu'à la faveur de la séduction trompeuse du langage (et des erreurs fondamentales de la raison qui y sont pétrifiées), lequel comprend, et comprend de travers, toute production d'effets comme conditionnée par une chose qui exerce ses effets, par un « sujet ». De même, en effet, que le peuple sépare la foudre de sa lueur et considère cette dernière comme un agir, comme effet exercé par un sujet qui s'appelle foudre, de même la morale du peuple sépare également la vigueur des extériorisations de cette vigueur comme s'il y avait derrière le vigoureux un substrat indifférent auquel il appartiendrait en toute liberté ou non d'extérioriser ou non sa vigueur. Mais un tel substrat n'existe pas ; il n'y a pas d' « être » derrière l'agir, la production d'effets, le devenir ; « l'agent » est purement et simplement ajouté de manière imaginative à l'agir - l'agir est tout. Fondamentalement, le peuple dédouble l'agir, quand il fait luire la foudre, c'est un agir d'agir : il pose le même événement d'abord comme cause et encore une fois ensuite comme son effet ${ }^{24}$.

La volonté de puissance, en s'exprimant par l'action des forts sur les faibles, ne constitue pas des sujets forts. Une telle représentation n'existe que dans l'esprit de ceux qui subissent la force. Le point de vue des faibles procède en reconstituant le fait de leur domination, ce qui revient à essentialiser un processus, à substantialiser le dynamisme de la force. La catégorie de responsabilité est ainsi appréhendée comme construction rétrospective, produite par le travail d'imputation auquel se livrent les faibles :

[...] Quoi d'étonnant si les affects rentrés de vengeance et de haine, couvant en secret, exploitent à leur profit cette croyance et si même, fondamentalement, il n'est pas de croyance qu'ils mettent plus d'ardeur à conforter que celle qui veut que le fort ait toute liberté d'être faible, et l'oiseau de proie d'être agneau : - ils s'attribuent ainsi le droit d'imputer à l'oiseau de proie la responsabilité d'être oiseau de proie... ${ }^{25}$

23. Généalogie de la morale, I, 13, p. 96.

24. Ibidem, p. 96-97.

25. Ibidem, p. 98-99. 
$\mathrm{Au}$ terme de ce fascinant raisonnement, la notion de responsabilité est donc envisagée comme une catégorie inventée de toutes pièces par les faibles pour désigner et accuser les forts de la « responsabilité »de leur propre force. Complexe, il signifie simultanément des choses différentes par leur portée. D'abord, on trouve ici l'écho de la tradition qui, partant des sophistes ${ }^{26}$ et arrivant à Spinoza ${ }^{27}$, en passant par Machiavel $^{28}$ et par Hobbes ${ }^{29}$, a illustré les rapports humains en se référant à un « droit de nature » pensable par référence à la férocité animale. On sait que la notion de volonté de puissance n'est en rien réductible à un « droit à la puissance », et que, telle que la concevait Nietzsche, elle ne correspond nullement à une volonté aspirant à la puissance, ou, contresens plus flagrant encore, à une volonté exprimant sa puissance (comme si la puissance était analytiquement contenue dans la volonté, alors que celle-ci n'est qu'une des expressions, ou plutôt une construction idéaliste platement anthropologique, de celle-là). Cependant, l'effet de choc engendré par l'argumentation nietzschéenne repose sur l'utilisation effective de la métaphore animale dans ce qu'elle a de moins humain afin de caractériser les relations humaines, si bien qu'il est nécessaire de saisir derrière cet effet ce que signifie réellement l'argumentation ${ }^{30}$. Il me semble qu'elle désigne le caractère asymétrique des relations humaines,

26. Cf. le célèbre fragment d'ANTIPHON, Diels-Kranz $n^{\circ}$ XLIV, concernant l'opposition entre la nature et la loi (sinon, le témoignage indirect de PLATON et de son personnage Calliclès dans Gorgias, 483d sq., à savoir le thème de la supériorité physique et sociale des « forts » selon les passions).

27. SPINOZA, Traité théologico-politique, chapitre XVI : «Par droit et institution de la nature, je n'entends autre chose que les règles de la nature de chaque individu, selon lesquelles nous concevons chaque être comme déterminé naturellement à exister et à agir de façon précise. Par exemple, les poissons sont déterminés par la nature à nager, les gros à manger les petits, et c'est donc par un droit naturel souverain que les poissons sont maitres de l'eau et que les gros poissons mangent les petits », trad. fr. J. Lagrée et P.-F. Moreau, Paris, P.U.F., 1999, p. 505.

28. MachIAVEL, Le Prince, par exemple chapitre XVIII : la triple référence au centaure, au renard et au lion (dans MACHIAVEL, De Principatibus/Le Prince, trad. J.-L. Fournel et J.-Cl. Zancarini, Paris, P.U.F., 2000, p. 150-151), et la «ferocità» de Cesare BORGIA dans le chapitre VII, p. 88-89). Cf. également dans la galerie de portraits des empereurs romains qui constitue en partie le chapitre XIX du Prince, la figure de Sévère, un des seuls qui mérite d'être regardé comme un modèle à imiter, est qualifié de « lion très féroce et renard très malin $»$, p. 166-167.

29. Cf. la thèse de l'homme «loup pour l'homme », dans HoBBEs, De cive, épître dédicatoire au comte de Devonshire, trad. Sorbière éditée par S. Goyard-Fabre, Paris, Flammarion, 1982, p. 83.

30. Le rapport homme-animal dans une pensée telle que celle de Nietzsche dépasse évidemment le cadre de cette étude. Dans un article très suggestif, Blaise Benoît propose d'appréhender les relations homme-animal chez Nietzsche en tant qu'« interprétations immanentes à la réalité comme jeu pulsionnel en devenir », de sorte à les apercevoir comme «métaphores ou transposition l'un de l'autre». Cf. Blaise BENOIT, « Favoriser l'envol de la «bête blonde » (Nietzsche, l'homme, l'animal, et le surhumain)», dans Jean-Luc Guтснет (dir.), Usages politiques de l'animalité, Paris, L'Harmattan, p. 183-201. 
et se comprend dans le registre de ce que j'ai nommé ailleurs une «anthropologie de la férocité $»^{31}$. Cette conception de la condition humaine semble effectivement appréhender les relations entre individus en fonction des rapports que les espèces animales entretiennent spontanément, en particulier sur le mode de la prédation - cette métaphore présente le grand avantage d'imposer immédiatement la représentation d'une asymétrie entre les êtres naturels. Une telle approche sous-évalue toutefois les ressources de l'anthropologie de la férocité, laquelle s'affirme de manière plus fondamentale dans le triple registre de l'acceptation par l'individu de ses «pulsions » naturelles (acceptation qu'il est nécessaire de concevoir en termes de grande santé), de son assomption à ce qui arrive (fortuna machiavélienne ou amor fati nietzschéen), et d'une évaluation critique des mœurs dont il est contemporain. L'anthropologie de la férocité qualifie de ce fait une conception aristocratique de l'existence, et par suite elle détermine un certain rapport à la vie collective, qui ne se réduit nullement au désir de dominer les êtres aussi incapables de ressentir en eux la véritable puissance de la nature que de mettre leur existence en péril, ainsi que le commandent pourtant la fortuna ou le fatum.

Ensuite, si son argumentation concède que les faibles sont bel et bien la proie des forts, Nietzsche n'a en vue ni l'établissement ni la reconnaissance d'un quelconque droit de ceux-ci à disposer de ceux-là. La portée de son argumentation réside dans la défense des forts contre la stratégie insidieuse des faibles, dont le raisonnement repose sur un contre-sens fondamental: il est rigoureusement impossible d'imputer ses propres actes au fort qui s'exprime par la volonté de puissance, car cela revient à constituer une fiction illusoire, la subjectivité, laquelle serait capable de répondre de la volonté de puissance, à savoir de la justifier et de la maîtriser. Ce contre-sens repose sur une inversion de l'ordre des choses, en faisant de la volonté de puissance l'attribut de la subjectivité, quelque chose comme la force du sujet, tandis que ce dernier n'est en réalité que l'effet de la volonté ${ }^{32}$. De plus, du point de vue des forts par lesquels s'exprime la volonté de puissance, une telle désignation-accusation n'a aucun sens : il leur semble vain d'entreprendre de légitimer leur propre surabondance de forces devant les faibles accusateurs (s'ils le faisaient, ils cesseraient d'ailleurs d'être forts).

Le lecteur, enfin, est incité à poursuivre le raisonnement de Nietzsche, à en comprendre la portée grâce à la reconstitution des motivations qui animent les faibles : sans doute déploient-ils une telle straté-

31. Je renvoie le lecteur à l'analyse que j'ai consacrée à cette thématique : Thierry MENISSIER, Machiavel ou la politique du centaure, Paris, Hermann, 2010, chapitre VIII, « L'anthropologie de la férocité », p. 297-339.

32. Pour une analyse convergente de la volonté de puissance dans ses relations avec la subjectivité, cf. Par-delà Bien et Mal, § 19, p. 31-32. 
gie dans le but de se défendre des agressions possibles des forts à leur encontre, mais peut-être que tel n'est pas leur mobile décisif. En accusant les forts, les faibles espèrent maitriser la volonté de puissance. Pourtant leur faiblesse dément un tel espoir, si l'on entend par là une captation de la force par les faibles; en effet, une « appropriation faible » de volonté de puissance ruinerait-elle la puissance de la volonté ? La véritable stratégie des faibles se révèle alors : l'imputation de la responsabilité de la volonté de puissance aux forts ainsi que la mise en accusation de cette dernière, conjuguées à son impossible appropriation par les faibles, ont pour effet de la dégrader irrémédiablement. Le véritable danger que fait peser sur la volonté de puissance l'imputation de responsabilité est de ruiner toute manifestation de la force.

Se faire le défenseur de «l'irresponsabilité des forts», en dénonçant les motivations réelles des accusateurs de la volonté de puissance, et en suggérant les énormes dangers que fait courir à l'espèce une telle stratégie, conduit Nietzsche à adopter une posture héroïque, à assumer d'être quelque chose comme le sauveur de la volonté de puissance, dans une argumentation difficilement justifiable auprès du grand public qu'il estimerait acquis à la thèse de la faiblesse.

\section{CONCLUSION}

Nous devons conclure de cette enquête que la philosophie nietzschéenne restituée dans son dessein mène à une réfutation de la notion de responsabilité, entendue à la fois comme contrôle conscient qu'un individu peut avoir sur les actes qui lui sont imputables et comme reconnaissance de la nécessité de ce contrôle en vue de la possibilité d'une vie collective. Comment vivrait concrètement un individu se comportant de la sorte ? À cette question, Nietzsche ne donne jamais de réponse univoque ni complète, mais à plusieurs reprises dans son œuvre, il sème des indices permettant d'en avoir une idée. On pourrait ainsi affirmer que le comportement de l'individu vivant au-delà du régime normal de la responsabilité est caractérisé par cette forme d'innocence très difficile à acquérir de la part d'un homme : la légèreté de Zarathoustra, le dieuhomme, à la figure duquel la fin du second traité fait appel ${ }^{33}$. Si l'on se tourne vers le poème de Zarathoustra, on apprend que, tandis que la volonté est capable de devenir «malfaitrice », en s'infligeant l'esprit de vengeance qui débouche sur le fait de châtier l'existence, elle est aussi susceptible, en étant créatrice, d'être un pouvoir de libération par rap-

33. Généalogie de la morale, II, $\S 25$, p. 179 : «- Mais que suis-je en train de dire? Assez! Assez! À ce point, il n'y a pour moi qu'une attitude convenable, me taire : je porterais la main sur ce qui n'est permis qu'à un plus jeune, à un plus «à venir », à un plus fort que moi, - ce qui n'est permis qu'à Zarathoustra, Zarathoustra le sans-dieu... » 
port aux entraves de la morale ${ }^{34}$. Tout le problème qui se pose alors est de mettre l'individualité au niveau des vertus de la guerre, cette grande vectrice de liberté 35 .

L'entreprise nietzschéenne de généalogie critique de la responsabilité accouche donc de la promesse d'une vie sur-humaine. Comment entendre cette dernière ? On pourrait proposer cette perspective : les individus capables d'échapper à l'assujettissement que représente la responsabilité-culpabilité n'imaginent jamais devoir rendre des comptes à autrui, et comme ils ne sauraient souscrire à l'égalitarisme impliqué par la reconnaissance de la loi commune, leurs actes ne sauraient pas même être jugés par les autres. Sans doute vivent-ils la relation avec autrui sur le mode des astres qui se croisent, ainsi que le suggère métaphoriquement le magnifique aphorisme du Gai savoir intitulé «Amitié d'astres » :

Amitiés d'astres. - Nous étions amis et nous sommes devenus étrangers l'un à l'autre. Mais il est bon qu'il en soit ainsi, et nous ne chercherons pas à nous le dissimuler ni à l'obscurcir comme si nous devions en avoir honte. Tels deux navires dont chacun poursuit sa voie et son but propres : ainsi sans doute nous pouvons nous croiser et célébrer des fêtes entre nous comme nous l'avons déjà fait - et alors les bons navires reposaient côte à côte dans le même port, sous le soleil, si calmes qu'on eût dit qu'ils fussent déjà au but et n'eussent eu que la même destination. Mais ensuite l'appel irrésistible de notre mission nous poussait à nouveau loin l'un de l'autre, chacun sur des mers, vers des parages, sous des soleils différents - peut-être pour ne plus jamais nous revoir, peut-être aussi pour nous revoir une fois de plus, mais sans plus nous reconnaitre : des mers et des soleils différents ont dû nous changer! Que nous dussions devenir étrangers l'un à l'autre, tel le voulait la loi au-dessus de nous : c'est par là même que nous devons devenir l'un pour l'autre plus respectable ! C'est par là même que la pensée de notre amitié d'autrefois doit nous être plus sacrée! Il est probablement une immense courbe invisible, une immense voie stellaire nos routes et nos buts où divergents se trouvent inscrits comme d'infimes trajets - élevons-nous à cette pensée ! Mais notre vie est trop brève, notre vision trop faible pour que nous puissions être davantage que des amis au sens de cette possibilité su-

34. Ainsi parlait Zarathoustra, II, « De la rédemption », op. cit. p. 175-180; cf. également à propos du caractère créateur de la volonté, ibidem, II, « Aux îles fortunées », p. 111-112, et III, « De la rapetissante vertu », p. 213.

35. Et de ce point de vue, on comprend les considérations qui, dans le passage du Crépuscule des idoles cité plus haut, font suite à la définition de la liberté comme « volonté de répondre de soi »: «[La liberté] C'est maintenir les distances qui nous séparent. C'est être indifférent aux chagrins, aux duretés, aux privations, à la vie même. C'est être prêt à sacrifier les hommes à sa cause, sans faire exception de soi-même. Liberté signifie que les instincts virils, les instincts joyeux de guerre et de victoire, prédominent sur tous les autres instincts, par exemple sur ceux de « bonheur », etc. », trad. citée, p. 156. 
blime ! - Et ainsi nous voulons croire à notre amitié d'astres, dussions-nous être ennemis sur la terre ${ }^{36}$.

Les astres se reconnaissent entre eux, dans leur solitude, et ils dérogent à tous les modes de la responsabilité qu'il est possible de mettre en valeur : responsabilité comme capacité et volonté personnelle de légitimer ses actes devant autrui ; comme prise en charge d'autrui dans une relation d'aide et de direction ; comme reconnaissance de la valeur supérieure de la loi civile commune ; comme acceptation de la loi morale en tant que critère de détermination de la volonté morale ${ }^{37}$. Cette multiple dérogation apparaît telle une disposition bien difficile à tenir, dessinant de ce fait le profil d'un ethos fort élitiste. Mais dans cette disposition partagée, une communauté paraît paradoxalement possible, ainsi que le suggère la lettre de ce texte littéralement saturé par la première personne du pluriel, tournure rarement employée par Nietzsche avec une telle intensité.

Pourtant, une remarque supplémentaire s'impose, au moment d'achever ce développement : second (et ironique) paradoxe, la nécessité reconnue par Nietzsche, pour parvenir à une telle vie relationnelle, de « surmonter l'homme » et de faire advenir le « surhomme ${ }^{38}$, signale assurément que le registre de la responsabilité fait profondément partie de la condition humaine. $\begin{array}{ll}\text { Université Pierre Mendès France } & 1251 \text { Avenue Centrale, } \\ \text { Département de philosophie } & \text { BP 47, 38040 Grenoble }\end{array}$ Département de philosophie

36. Le gai savoir, « Amitiés d'astres », § 279, trad. fr. P. Klossowski, Paris, Gallimard, 1967, revue et augmentée par M. de Launay, 1982, p. 191-192.

37. Cf. également Par-delà Bien et Mal, § 29, à propos de «l'indépendance des forts », et 44, à propos de la solitude des « esprits libres.

38. Cf. Ainsi parlait Zarathoustra, III, « De l’homme supérieur », p. 346-357. 
Resume. - Généalogie, critique de la responsabilité morale et constitution de la subjectivité selon Nietzsche. By Thierry MENISSIER.

Dans cet article, on s'attache à restituer la critique nietzschéenne de la notion de responsabilité morale, à partir d'une lecture des thèses de la Généalogie de la morale. Cette critique met en relief le style de l'entreprise généalogique; elle révèle en quoi la subjectivité, dans l'optique nietzschéenne, repose sur la structure de la dette et permet de comprendre ce que toute construction de responsabilité doit à la mauvaise conscience. La critique de la responsabilité jette de plus un éclairage saisissant sur les mécanismes de dressage qui constituent la culture. Il s'agit ensuite de s'interroger sur la portée des conséquences de la critique nietzschéenne, en réfléchissant au profil des subjectivités débarrassées de la culpabilité telles que les appelle l'auteur de la Généalogie de la morale (mutation ou abolition de la notion de responsabilité ?).

MOTS-CLEFS : Nietzsche - responsabilité - généalogie de la morale - subjectivité faute-culture occidentale.

AbSTRACT. - Genealogy, the critique of moral responsibility and the constitution of subjectivity in Nietzsche. By Thierry MENISSIER.

This article attempts to reconstruct Nietzsche's critique of the notion of moral responsibility, based on a reading of the ideas contained in the Genealogy of Morals. This critique focuses on the stylistic aspect of Nietzsche's genealogical enterprise; it reveals how, from a Nietzschean perspective, subjectivity is founded upon the structure of debt, enabling us to understand what every configuration of responsibility owes to the bad conscience. Moreover, the critique of responsibility sheds a striking light upon the training mechanisms that make up culture. The significance of the consequences of the Nietzschean critique is subsequently examined by considering the profile of types of subjectivity that are free from guilt, as described in the Genealogy of Morals (transformation or abolition of the notion of responsibility?)

KEY WORDS : Genealogy of morals - Nietzsche - responsibility - subjectivity transgression - western culture. 